\title{
Investigation and management of chronic cough using a probability-based algorithm
}

\author{
J.A. Kastelik, I. Aziz, J.C. Ojoo, R.H. Thompson, A.E. Redington and A.H. Morice
}

ABSTRACT: Chronic cough is a common and distressing symptom. A novel algorithm has been developed for the management of chronic cough, in which an assessment of clinical probability of disease determines the need to proceed to investigation.

In this study, the performance of this algorithm in clinical practice was prospectively evaluated. A total of 131 consecutively referred patients ( 86 females) whose principal presenting symptom was a cough of duration $>8$ weeks were studied. Their age (median (range)) was 60 (16-88) yrs and cough duration $5.9(0.2-65)$ yrs.

A cause of cough was established in $93 \%$ of cases. The most frequent diagnoses were asthma ( $24 \%$ of cases), gastro-oesophageal disease (22\%), post-viral cough ( $8 \%)$, bronchiectasis $(8 \%)$ and interstitial lung disease $(8 \%)$. Primary pulmonary disease was significantly more likely in patients with a productive cough and in patients with an abnormal chest radiograph. Only a small proportion ( $<8 \%$ ) of patients had multiple causes of cough. The probability of treatment started on the basis of a high clinical suspicion of either asthma, gastro-oesophageal disease or rhinitis being successful was $74 \%$. Overall, $26 \%$ of the patients were managed successfully without the need for any form of investigation other than chest radiography and spirometry.

Use of the algorithm resulted in identification of the cause of cough and successful treatment in the large majority of cases. It is concluded that this protocol has the potential to improve management by providing a structured approach, reducing the number of investigations performed, and minimising unnecessary delays in treatment.

KEYWORDS: Asthma, cough, diagnosis, treatment

hronic cough is a common and distressing symptom [1, 2]. Historically, a number of approaches have been taken for the diagnosis and management of patients with this complaint, including an anatomical diagnostic protocol [3-10], full investigation for common causes in all patients [11, 12], and empirical trials of therapy without initial investigation [13-15]. The frequency with which the condition has been diagnosed and successfully treated has varied substantially between these studies. In general, higher success rates have been claimed when a systematic investigational protocol was used. A consensus panel report of the American College of Chest Physicians on the clinical management of cough was published in 1998 [16].

A novel algorithm has been developed for the investigation and management of chronic cough.

For editorial comments see page 213.
In contrast to previous approaches, this algorithm requires a clinical assessment of disease probability in order to determine whether to initiate treatment or investigate further. This algorithm has now been prospectively applied to a large group of patients referred to a specialist cough clinic. The present study demonstrates that this led to the identification of the cause of cough and successful treatment in a large majority of cases. The pattern of diagnoses reached, the predictive value of cough character and radiographic features, and the need for specialised investigation when assessing patients with chronic cough are reported.

\section{METHODS}

\section{Patients}

Consecutive adult patients seen as secondary or tertiary referrals to a specialist clinic at a university hospital (Hull Cough Clinic, Castle Hill Hospital, University of Hull, Hull, UK)

\section{AFFILIATIONS}

Division of Academic Medicine, Postgraduate Medical Institute, University of Hull, Hull, UK.

CORRESPONDENCE

A.H. Morice

Division of Academic Medicine Castle Hill Hospital

Castle Road

Cottingham

East Yorkshire HU16 5JQ

UK

Fax: 441482624068

E-mail: a.h.morice@hull.ac.uk

Received

December 192003

Accepted after revision:

September 262004
European Respiratory Journal Print ISSN 0903-1936 Online ISSN 1399-3003 


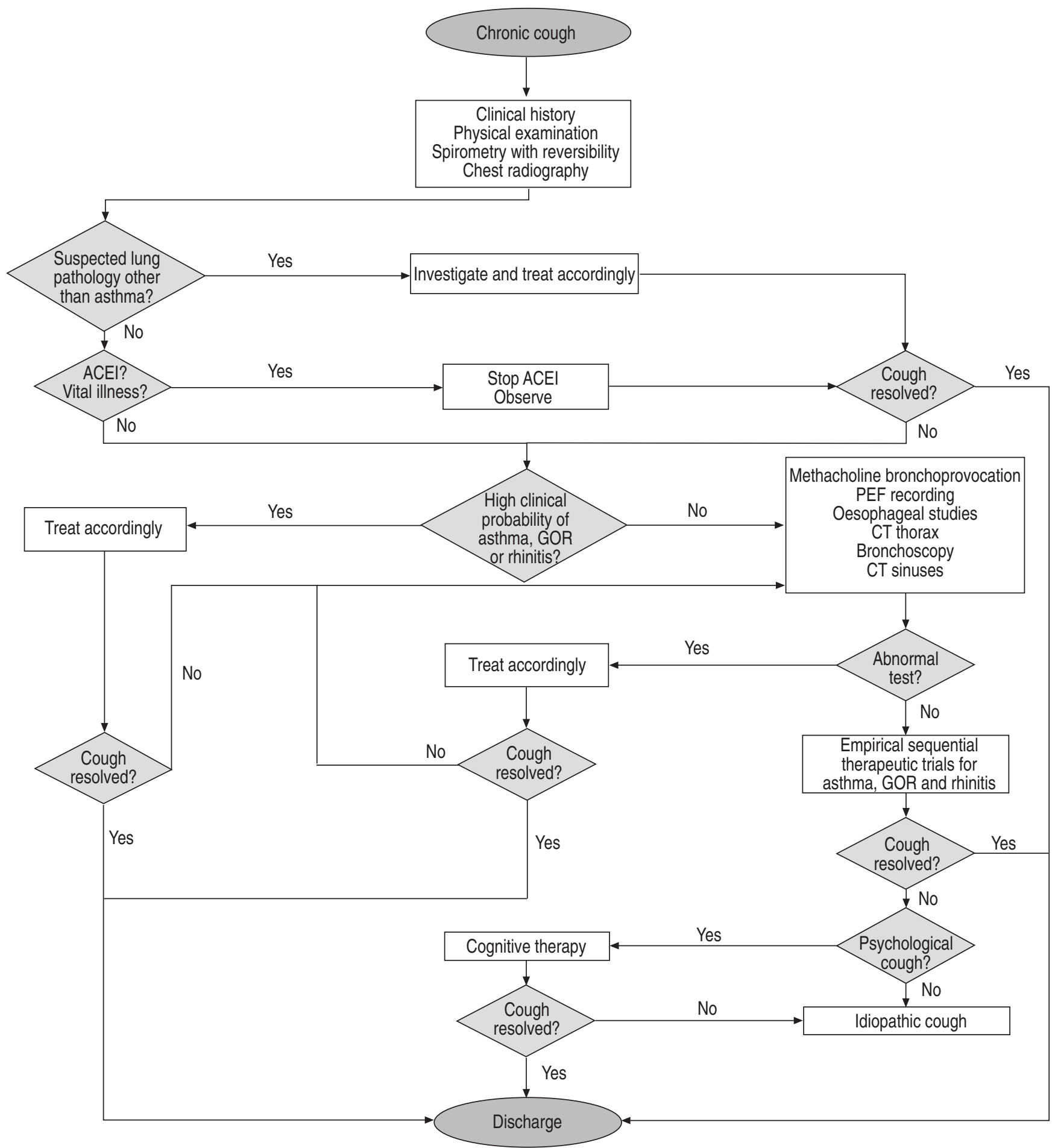

FIGURE 1. Algorithm for the investigation and management of chronic cough. ACEl: angiotensin-converting enzyme inhibitor; GOR: gastro-oesophageal reflux; PEF: peak expiratory flow; CT: computed tomography.

between August 1998 and April 2000 were studied. Their presenting symptom was chronic cough, defined as cough persisting for $>8$ weeks. All patients gave informed consent prior to investigative procedures.

\section{Probability-based algorithm}

Patients were investigated and managed using a novel probability-based algorithm (fig. 1). Initial assessment was by clinical history and physical examination. Emphasis was placed on features of gastro-oesophageal and upper and lower airway disease. In addition, a history of recent upper respiratory tract infection, treatment with angiotensin-converting enzyme (ACE) inhibitors or $\beta$-adrenoceptor antagonists, cigarette smoking and occupational history were determined. Chest radiography and spirometry, with bronchodilator reversibility if appropriate, were performed in all cases. 
The major components of the subsequent evaluation were as follows. 1) When the initial assessment suggested a primary lung pathology other than asthma, further investigations were arranged as indicated. 2) Patients with suspected post-viral cough or ACE inhibitor-induced cough at initial assessment were managed as below without additional investigation. 3) When there was a high clinical probability, as defined below, of asthma, gastro-oesophageal disease or rhinitis at initial assessment, appropriate treatment was started without further investigation. 4) In cases without a high clinical probability of these conditions, patients underwent sequential investigation. Patients who failed to respond to a trial of treatment initiated on the basis of a high clinical probability were also investigated. Treatment was commenced when results were abnormal. 5) When all investigational results were normal, empirical therapeutic trials were undertaken.

A final diagnosis was usually only accepted when patients reported that the symptom of cough had resolved, or had reduced to the extent that it was no longer troublesome, in response to therapy. An exception was in conditions such as bronchiectasis and interstitial lung disease, for which the nature of the disease reduced expectations of a major improvement.

\section{Diagnostic criteria and management}

Asthma

Reversible airflow obstruction and a history of episodic wheeze or breathlessness were all considered to indicate a high probability of asthma. When these features were absent, a diagnosis of asthma was pursued by peak expiratory flow (PEF) monitoring and/or methacholine bronchoprovocation. Treatments for asthma included short-acting $\beta_{2}$-agonists, inhaled corticosteroids (budesonide $400 \mu \mathrm{g} \cdot$ day $^{-1}$ or equivalent) and leukotriene receptor antagonists.

\section{Gastro-oesophageal disease}

A high clinical probability of gastro-oesophageal disease was suggested by a history of heartburn, dysphagia, or acid regurgitation, or an association between cough and posture or eating. In the absence of these features, oesophageal 24-h pH monitoring and manometry were carried out. Antireflux therapy included proton pump inhibitors such as lansoprazole $30 \mathrm{mg}$ daily or equivalent, alginates, and advice about diet and posture. Therapy was continued for $\geqslant 3$ months.

\section{Rhinosinusitis}

A high clinical probability of rhinosinusitis was suggested by symptoms of frequent throat clearing, postnasal drip, nasal discharge, nasal obstruction or sneezing, and when clinical examination of the nasopharynx and oropharynx revealed mucopurulent secretions or mucosal inflammation. In the absence of these criteria, a sinus computed tomography (CT) scan was performed. Management, in accordance with current guidelines [17], included topical intranasal corticosteroids, oral antihistamines and nasal decongestants, such as pseudoephedrine. In severe disease, referral was made to an otorhinolaryngologist for consideration of surgical intervention.

\section{Post-viral cough}

Post-viral cough was considered probable when the onset of cough showed a clear temporal relation to an episode of upper respiratory tract infection. Providing the initial investigations were normal, no additional investigations were performed. The final diagnosis was accepted if cough resolved within 2 months.

\section{Other pulmonary diseases}

Bronchiectasis was suggested by a history of expectoration of purulent sputum. The diagnosis was confirmed by compatible features on chest radiographs or high-resolution CT (HRCT) scans. Patients with bronchiectasis were treated with chest physiotherapy, antibiotics, bronchodilators and surgery for limited disease. Chronic obstructive pulmonary disease (COPD) was suggested by symptoms of progressive breathlessness and wheeze, a $\geqslant 20$ pack-yr history of cigarette smoking and airflow obstruction without bronchodilator reversibility. Management included smoking cessation measures and bronchodilators. Interstitial lung disease was initially suggested by clinical features and radiographic appearance. The diagnosis was confirmed by HRCT scanning, pulmonary function testing and, in selected cases, lung biopsy. Treatments included oral corticosteroids and immunosuppressive agents.

\section{Angiotensin-converting enzyme inhibitor-induced cough}

Patients taking ACE inhibitors were asked to discontinue these agents, and angiotensin II receptor antagonists were introduced in their place if appropriate. Providing that the results of initial investigations were normal, these patients did not undergo any further tests. The diagnosis was accepted if cough resolved after cessation of the ACE inhibitor.

\section{Idiopathic and psychogenic cough}

Cough was categorised as idiopathic only when the results of trials of treatment for asthma, gastro-oesophageal reflux and rhinosinusitis were all negative. When there was suspicion of a psychogenic cause on the basis of proposed diagnostic criteria [18], patients were referred for cognitive psychotherapy.

\section{Investigative procedures}

Spirometry and reversibility

Spirometry was performed using a Vitalograph compact spirometer (Vitalograph, Buckingham, UK). Subjects completed three forced expiratory manoeuvres from total lung capacity to residual volume, and the best value was used in the analysis. The forced expiratory volume in one second (FEV1), forced vital capacity (FVC) and FEV1/FVC ratio were recorded. Spirometry was repeated $15 \mathrm{~min}$ after inhalation of nebulised salbutamol $2.5 \mathrm{mg}$. Reversibility was defined as an increase in FEV1 of $\geqslant 15 \%$ of the pre-bronchodilator value and $>200 \mathrm{~mL}$. Airflow obstruction was defined as an FEV1/FVC ratio of $<70 \%$.

\section{Peak expiratory flow monitoring}

PEF was recorded twice daily as the best of three blows over a period of $\geqslant 2$ weeks. Diurnal PEF variability was assessed by visual inspection.

\section{Airway responsiveness}

Methacholine bronchoprovocation was performed according to European Respiratory Society guidelines [19], using an Aerosol Provocation System and MasterScope (Jaeger Toennies, Hochberg, Germany). The cumulative provocative 


\begin{tabular}{lc}
\hline TABLE 1 & Characteristics of patients studied \\
\hline Characteristics & \\
\hline Subjects $\mathbf{n}$ & 131 \\
Females & $86(66)$ \\
Age yrs & $60(16-88)$ \\
Duration of cough yrs & $5.9(0.2-65.0)$ \\
Current smokers & $6(5)$ \\
Dry cough & $51(39)$ \\
Abnormal chest radiograph & $49(37)$ \\
FEV 1 \% pred & $89 \pm 20$ \\
FVC \% pred & $93 \pm 19$ \\
Obstructive deficit on spirometry & $21(16)$ \\
Reversibility to salbutamol & $10(8)$ \\
\hline
\end{tabular}

Data are presented as $\mathrm{n}, \mathrm{n}(\%)$, median (range) and mean $\pm \mathrm{SD}$. FEV1: forced expiratory volume in one second; \% pred: percentage of the predicted value; FVC: forced vital capacity

dose of methacholine required to produce a $20 \%$ fall in FEV1 (PD20) was determined by computer-assisted linear interpolation from the logarithmic dose-response plot. Airway hyperresponsiveness was defined as a PD20 of $<500 \mu \mathrm{g}$.

\section{Oesophageal 24-h pH monitoring and manometry}

Ambulatory 24-h oesophageal $\mathrm{pH}$ monitoring was performed as previously described [20, 21], using a glass $\mathrm{pH}$ electrode (Mettler-Toledo, Leicester, UK) connected to a portable data storage unit (Digitrapper MKII Gold; Medtronic, Watford, UK). Gastro-oesophageal reflux was defined as occurring when a $\mathrm{pH}$ of $<4.0$ was recorded for $>4 \%$ of the total time during the 24-h period. Oesophageal manometry was performed with a 9-lumen radially orientated catheter (Mediplus, High Wycombe, UK) according to a previously described protocol [21], with motility analysis based on 10 wet swallows. Manometric results were considered abnormal when the number of nonconducted contractions was $\geqslant 30 \%$ of the total number of swallows in the wet swallow series, low-amplitude peristaltic contractions were $<15 \mathrm{~cm} \mathrm{H}_{2} \mathrm{O}$, the lower oesophageal sphincter pressure (abdominal component) was $<10 \mathrm{~cm}$ $\mathrm{H}_{2} \mathrm{O}$, or double-peaked, triple-peaked, simultaneous or tertiary contractions were present.

\section{Fibreoptic bronchoscopy}

When a mass lesion was suspected or prior to diagnosis of idiopathic cough, bronchoscopy was performed using a flexible fibreoptic bronchoscope (EB-1530T2; Pentax Optical Company, Tokyo, Japan). The nasal passages, pharynx, larynx and bronchial tree were inspected.

\section{Statistical analysis}

Data for age and cough duration were expressed as median (range) and for spirometric measurements as mean $\pm \mathrm{SD}$. Multiple logistic regression was performed to study the predictive value of cough character and abnormal chest radiography results, and exact $95 \%$ confidence intervals were calculated for probabilities. A p-value of $<0.05$ was regarded as significant.

\section{RESULTS}

In total, 148 patients were referred for assessment. However, eight failed to attend a follow-up appointment, two died due to unrelated causes, two moved from the area, and five declined further investigation or treatment. Therefore, full information was available for 131 patients, of whom 116 were referred by a primary care physician and 15 by a hospital specialist. Their clinical characteristics are summarised in table 1.

Using the algorithm, a final diagnosis was reached in $93 \%$ of patients (fig. 2). The conditions diagnosed and their relative frequencies are shown in table 2 . In 10 patients, cough was due to more than one cause; in these cases, only the primary diagnosis was used for the purpose of the analysis. Asthma and gastro-oesophageal disorders were the commonest causes of cough identified. Sixty-one (46\%) patients showed primary lung pathology, $29(22 \%)$ had primary gastro-oesophageal disease, and 19 (10\%) primary upper airways disorders. A diagnosis of primary pulmonary disease was significantly more likely in patients with a productive cough (OR $(95 \%$ confidence interval (CI)) of $3.7(1.5-9.5) ; \mathrm{p}=0.005)$ and those with an abnormal chest radiograph (7.7 (2.9-20.7); $\mathrm{p}<0.001)$. Neither cough character nor chest radiographic findings were predictive of a diagnosis of primary gastro-oesophageal disease. The numbers of patients with upper airway disease and in other diagnostic categories were too few to allow meaningful statistical analysis.

The probabilities of successful management at each major point in the algorithm are shown in table 3. A primary lung pathology other than asthma (bronchiectasis, interstitial lung disease, COPD or lung neoplasm) was suspected at the initial assessment in 29 cases, and confirmed by additional investigation, as appropriate, in 26 of these. In the other three cases, bronchiectasis had been suspected, but this diagnosis was not supported by HRCT scanning. In the six patients receiving an ACE inhibitor, cough improved within 3-8 weeks from withdrawal of this agent. Cough resolved spontaneously in all of the 11 cases of suspected post-viral cough.

Treatment started on the basis of a high clinical probability of asthma, gastro-oesophageal disease or rhinosinusitis was successful in 17 of 23 patients, with no additional investigation required. The probability $(95 \% \mathrm{CI})$ of successful management in this circumstance was therefore $74 \%$ (52-90). The 17 cases accounted for seven (22\%) of the 32 diagnoses of asthma, seven $(24 \%)$ of the 29 diagnoses of gastro-oesophageal disease and three $(38 \%)$ of the eight diagnoses of rhinitis. Including the six patients with ACE inhibitor-induced cough and the 11 with post-viral cough, therefore, $34(26 \%)$ of the 131 patients studied were managed successfully without any additional investigation.

A total of 65 patients with a low clinical probability of asthma, gastro-oesophageal reflux or rhinosinusitis underwent one or more investigations. Investigations were also performed in six patients who showed high pre-test probabilities of either rhinitis $(n=3)$, gastro-oesophageal reflux $(n=2)$ or asthma $(n=1)$, but failed to respond to therapeutic trials for these conditions. A cause of cough was established in 50 of these 71 


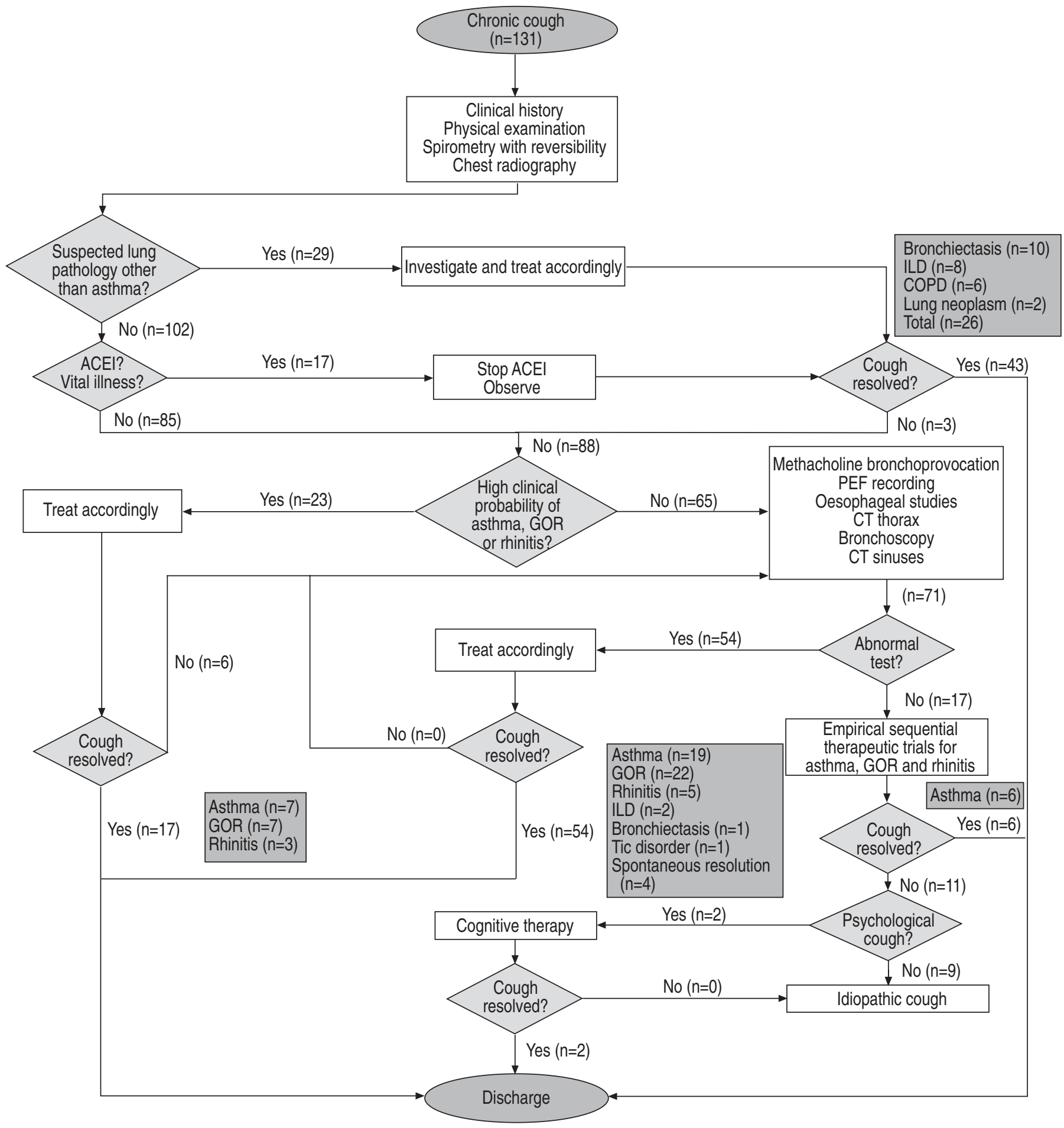

FIGURE 2. Annotated algorithm showing numbers of patients and diagnoses reached. ILD: interstitial lung disease; COPD: chronic obstructive pulmonary disease; ACEl: angiotensin-converting enzyme inhibitor; GOR: gastro-oesophageal reflux; PEF: peak expiratory flow; CT: computed tomography.

cases, on the basis of both an abnormal test and a subsequent response to a therapeutic trial. Spontaneous improvement occurred in a further four cases. The overall probability $(95 \%$ $\mathrm{CI})$ of successful management of these patients was therefore $76 \%(64-85)$. One patient was felt to have a tic disorder; details have been reported previously [22]. The investigations performed and their outcomes are summarised in table 4 .
All investigational results were normal in the 17 patients who subsequently underwent sequential therapeutic trials. Cough resolved in only six cases, and the probability of successful management was therefore relatively low at 35\% (14-62). Two patients were considered to have psychogenic cough. In the remaining nine cases, no definitive diagnosis could be reached and the cough was categorised as idiopathic. Of these, three 


\begin{tabular}{lc}
\hline TABLE 2 & $\begin{array}{l}\text { Causes of chronic cough in order of decreasing } \\
\text { frequency }\end{array}$ \\
\hline Diagnosis & Patients \\
\hline Asthma & $32(24)$ \\
Gastro-oesophageal disorders & $29(22)$ \\
Post-viral cough & $11(8)$ \\
Bronchiectasis & $11(8)$ \\
Interstitial lung disease & $10(8)$ \\
Idiopathic cough & $9(7)$ \\
Rhinosinusitis & $8(6)$ \\
COPD & $6(5)$ \\
ACE inhibitor therapy & $6(5)$ \\
Spontaneous resolution & $4(3)$ \\
Lung neoplasm & $2(1.5)$ \\
Psychogenic cough & $2(1.5)$ \\
Tic disorder & $1(0.5)$ \\
\hline
\end{tabular}

Data are presented as $n(\%)$. COPD: chronic obstructive pulmonary disease; ACE: angiotensin-converting enzyme.

patients showed clinical features of rhinitis but failed to respond to treatment, three had suspected asthma but responded only partially to oral corticosteroids and two had abnormal oesophageal study results but persistent cough despite antireflux therapy. In one case, all test results were normal and cough persisted despite therapeutic trials.

\section{DISCUSSION}

In the present study, a novel algorithm for the investigation and management of chronic cough was evaluated in a large unselected group of patients. A central feature differentiating this from previous strategies was the assessment made of clinical probability of disease. Thus, when there was a high probability of asthma, gastro-oesophageal reflux or rhinosinusitis, patients proceeded immediately to therapeutic trials. In contrast, when the clinical probability of these disorders was not high, patients were investigated and treatment was started on the basis of abnormal results. In some respects, this might be seen as analogous to the approach that has been taken in the diagnosis of venous thromboembolism, where stratification by pre-test probability of disease has been found helpful in

\section{TABLE 4 Investigations performed and proportion of} abnormal results

\begin{tabular}{lc} 
Investigation & Procedures \\
\hline & $76(25)$ \\
Methacholine challenge & $46(59)$ \\
Thoracic CT scan & $33(67)$ \\
24-h oesophageal pH monitoring and manometry & $27(15)$ \\
Fibreoptic bronchoscopy & \\
\hline
\end{tabular}

Data are presented as n (\% abnormal). CT: computed tomography.

interpreting investigational results and establishing a final diagnosis [23].

Using the present protocol, it was found that an underlying cause could be established in $>90 \%$ of patients with chronic cough. The pattern of diagnoses reached, however, differed from that in several previously published studies. In particular, it was found that asthma, gastro-oesophageal reflux and rhinitis were present together in only about half of the patients. This contrasts with reports that these three diagnoses represent the commonest causes of chronic cough, accounting for up to $90 \%$ of cases in some series $[3,5,7,8,11]$. Conversely, it was found that other forms of pulmonary disease (mainly bronchiectasis, interstitial lung disease and COPD) explained $>20 \%$ of cases, and that post-viral cough and ACE inhibitor therapy were also relatively common. These differences emphasise the fact that the pattern of diagnoses in chronic cough varies with the referral population. Many previous series, for example, have excluded patients with chest radiographic abnormalities or significant smoking histories [6, 1012]. However, the present unselected study may be more representative of the population referred for opinion to secondary care. Only a small proportion $(<8 \%)$ of patients with multiple causes of chronic cough were identified, consistent with other reports that cough is due to a single cause in $89-100 \%$ of cases $[6,9,10]$. Cough was categorised as idiopathic on only nine occasions.

Cough was successfully treated in the large majority of patients in the present study. In previously reported series of patients with chronic cough, response to treatment has varied greatly. Centres using a systematic protocol have reported figures in the range of $82-100 \%$ of cases [3-11], whereas almost

TABLE 3 Probability of successful management at each major point in the algorithm

\begin{tabular}{lcc} 
& Patients & Probability \\
\hline Suspected lung pathology other than asthma & 29 & $90(73-98)$ \\
Suspected post-viral cough & 11 & $100(72-100)$ \\
Suspected ACE inhibitor-induced cough & 6 & $100(54-100)$ \\
High clinical probability of asthma, GO disease or rhinosinusitis & 23 & $74(52-90)$ \\
Low clinical probability of asthma, GO disease or rhinosinusitis (or failed & 54 & $76(64-85)$ \\
$\quad$ therapeutic trial) & 17 & $35(14-62)$ \\
\hline
\end{tabular}

Data are presented as $\mathrm{n}$ and \% (95\% confidence interval). ACE: angiotensin-converting enzyme; GO: gastro-oesophageal. 
$50 \%$ of patients were treated without success in surveys from clinics that did not apply a systematic approach $[13,24]$. In the context of chronic cough, precise quantification of response is difficult as the definition of therapeutic success in all studies, including the present one, has been subjective and relatively imprecise. Measurements of cough reflex sensitivity [25] and quality of life $[1,26,27]$ have previously been used to provide support for therapeutic success, but the development of a reliable and clinically useful cough monitor would represent a significant advance.

It was found that the clinical assessment of disease probability markedly assisted management. Treatment started on this basis was successful on $74 \%$ of occasions, and these patients required no further investigation. Similarly, a history of either ACE inhibitor treatment or a preceding viral illness were both highly predictive of resolution of cough with appropriate management. Over a quarter of the patients were therefore diagnosed and successfully managed without any form of investigation other than a plain chest radiograph and simple spirometry. This approach has the potential to minimise delays in starting appropriate treatment, and also to avoid the expense and possible adverse effects associated with unnecessary investigation.

Eosinophilic bronchitis is a relatively recently recognised condition, characterised by chronic cough and sputum eosinophilia in the absence of variable airflow obstruction or airway hyperresponsiveness [28, 29]. It has been reported that eosinophilic bronchitis may account for up to $10-15 \%$ of patients attending cough clinics [10]. In the present study, sputum induction was not performed and it would, therefore, not have been possible to diagnose eosinophilic bronchitis. However, all patients with normal bronchoprovocation test and spirometry results, and with no other cause of cough identified, underwent a trial of inhaled or oral corticosteroids. This approach, therefore, permitted the detection of corticosteroid-responsive cough, of which eosinophilic bronchitis is one example. In the present series, only six such patients with normal airway responsiveness showed a positive response to anti-asthma therapy, suggesting that eosinophilic bronchitis may be relatively uncommon in the present population of patients. Nevertheless, these findings confirm that normal airway responsiveness does not preclude an improvement in cough with corticosteroids.

Rhinosinusitis was less common ( $6 \%$ of cases) in the present study than in several previous series [3,5-8]. It is unlikely that this was due to a failure to diagnose the condition, as the present protocol included systematic upper airway assessment together with a therapeutic trial. Diagnostic confusion may arise because the symptoms of nasal and postnasal disease are common in the general population and because conventional treatment for rhinosinusitis is nonspecific. For example, antihistamines administered unselectively to patients with chronic cough may lead to improvement through direct antitussive activity [30]. Furthermore, rhinitis is an independent risk factor for asthma [31], and is associated with both airway hyperresponsiveness [32] and lower airways inflammation [33], even in the absence of asthmatic symptoms. Therefore, antihistamines may exert an effect on unrecognised asthma, a condition in which antihistamines have established antitussive properties [34, 35].

It was found that cough character can be helpful in determining the aetiology of chronic cough. Specifically, a history of sputum production was predictive of a primary pulmonary cause. This contrasts with the findings of MeLLo et al. [8], who reported that cough character was not likely to be diagnostically useful in chronic cough. However, the complex regression model that these authors used, designed to explore whether the variability in multiple parameters of cough timing and character could be explained by a single specific diagnosis, may have obscured any such associations. The present analysis, in contrast, was confined to two predictor variables and a simple categorisation of site of disease.

An abnormal chest radiograph was also predictive of a primary pulmonary cause of chronic cough in the present study. Previous examinations of the relationship between diagnosis and radiographic findings have yielded conflicting results. PuOlijoKi and LAHDEnsuo [24], for example, found almost no diagnostic overlap between cases in which the chest radiograph was normal and those in which it was abnormal. In contrast, IRWIN et al. [5] reported that, compared with several other investigations, chest radiographs were much less frequently helpful. To the present authors' knowledge, the current study is the first to estimate the relative risk of pulmonary disease associated with an abnormal chest radiograph. Thoracic CT scanning proved useful in the diagnosis of pulmonary diseases, principally bronchiectasis and interstitial lung disease. This investigation was usually requested to confirm diagnoses suspected on the basis of plain chest radiographs, but there were three cases in which the chest radiograph appeared normal but the thoracic CT scan was abnormal. In previous series that did not include thoracic CT in the evaluation protocol, conditions such as these were seldom diagnosed as causes of chronic cough $[3,5,7]$. Conversely, indiscriminate use of CT produces a low diagnostic yield [12] and is unlikely to be cost-effective. The present authors suggest that thoracic CT scanning be performed in selected patients, particularly those with productive cough, even when the chest radiograph is normal.

In the present study, females outnumbered males by almost $2: 1$, consistent with most previous reports $[4,5,7-9,11,12,15]$. The reason for this marked sex difference in adults attending cough clinics is uncertain. However, increased cough reflex sensitivity has been described in female patients with chronic cough [36], as well as in female healthy volunteers [37, 38]. Similarly, ACE inhibitor-induced cough is reported more frequently in females [39]. These observations suggest an influence of hormonal status on cough reflex sensitivity, and this is further supported by a report that cough reflex sensitivity is similar in male and female pre-pubertal children [40].

In conclusion, experience with a refined diagnostic algorithm for chronic cough based on an assessment of clinical probability and selective use of investigations is reported. It has been shown that use of this algorithm led to the identification of the cause of cough and successful treatment in the large majority of cases. By providing a systematic 
structured approach, the authors believe that this protocol has the potential to improve management. It may also be economically advantageous in that it reduces the number of investigations performed, although economic outcomes were not directly evaluated. Finally, it should minimise unnecessary delays in treatment, and its use in primary care may obviate the need for referral to specialist services.

\section{REFERENCES}

1 French CL, Irwin RS, Curley FJ, Krikorian CJ. Impact of chronic cough on quality of life. Arch Intern Med 1998; 158: 1657-1661.

2 Morice AH, Kastelik JA. Chronic cough in adults. Thorax 2003; 58: 901-907.

3 Irwin RS, Corrao WM, Pratter MR. Chronic persistent cough in the adult: the spectrum and frequency of causes and successful outcome of specific therapy. Am Rev Respir Dis 1981; 123: 413-417.

4 Poe RH, Harder RV, Israel RH, Kallay MC. Chronic persistent cough. Experience in diagnosis and outcome using an anatomic diagnostic protocol. Chest 1989; 95: 723-728.

5 Irwin RS, Curley FJ, French CL. Chronic cough. The spectrum and frequency of causes, key components of the diagnostic evaluation, and outcome of specific therapy. Am Rev Respir Dis 1990; 141: 640-647.

6 Hoffstein V. Persistent cough in nonsmokers. Can Respir J 1994; 1: 40-47.

7 Smyrnios NA, Irwin RS, Curley FJ. Chronic cough with a history of excessive sputum production. The spectrum and frequency of causes, key components of the diagnostic evaluation, and outcome of specific therapy. Chest 1995; 108: 991-997.

8 Mello CJ, Irwin RS, Curley FJ. Predictive values of the character, timing, and complications of chronic cough in diagnosing its cause. Arch Intern Med 1996; 156: 997-1003.

9 Marchesani F, Cecarini L, Pela R, Sanguinetti CM. Causes of chronic persistent cough in adult patients: the results of a systematic management protocol. Monaldi Arch Chest Dis 1998; 53: 510-514.

10 Brightling CE, Ward R, Goh KL, Wardlaw AJ, Pavord ID. Eosinophilic bronchitis is an important cause of chronic cough. Am J Respir Crit Care Med 1999; 160: 406-410.

11 McGarvey LPA, Heaney LG, Lawson JT, et al. Evaluation and outcome of patients with chronic non-productive cough using a comprehensive diagnostic protocol. Thorax 1998; 53: 738-743.

12 Palombini BC, Villanova CAC, Araújo E, et al. A pathogenic triad in chronic cough: asthma, postnasal drip syndrome, and gastroesophageal reflux disease. Chest 1999; 116: 279-284.

13 McGarvey LPA, Heaney LG, MacMahon J. A retrospective survey of diagnosis and management of patients presenting with chronic cough to a general chest clinic. Int J Clin Pract 1998; 52: 158-161.

14 Pratter MR, Bartter T, Akers S, DuBois J. An algorithmic approach to chronic cough. Ann Intern Med 1993; 119: 977-983.
15 Simpson G. Investigation and management of persistent dry cough. Thorax 1999; 54: 469-470.

16 Irwin RS, Boulet L-P, Cloutier MM, et al. Managing cough as a defense mechanism and as a symptom. A consensus panel report of the American College of Chest Physicians. Chest 1998; 114: Suppl. 2, 133S-181S.

17 Bousquet J, Van Cauwenberge P, Khaltaev N, Aria Workshop Group, World Health Organization. Allergic rhinitis and its impact on asthma. J Allergy Clin Immunol 2001; 108: Suppl. 5, S147-S334.

18 Mastrovich JD, Greenberger PA. Psychogenic cough in adults: a report of two cases and review of the literature. Allergy Asthma Proc 2002; 23: 27-33.

19 Sterk PJ, Fabbri LM, Quanjer PH, et al. Airway responsiveness. Standardized challenge testing with pharmacological, physical and sensitizing stimuli in adults. Eur Respir J 1993; 6: Suppl. 16, 53-83.

20 Kastelik JA, Jackson W, Davies TW, et al. Measurement of gastric emptying in gastroesophageal reflux-related chronic cough. Chest 2002; 122: 2038-2041.

21 Kastelik JA, Redington AE, Aziz I, et al. Abnormal oesophageal motility in patients with chronic cough. Thorax 2003; 58: 699-702.

22 Ojoo JC, Kastelik JA, Morice AH. A boy with a disabling cough. Lancet 2003; 361: 674.

23 Perrier A, Desmarais S, Miron M-J, et al. Non-invasive diagnosis of venous thromboembolism in outpatients. Lancet 1999; 353: 190-195.

24 Puolijoki H, Lahdensuo A. Causes of prolonged cough in patients referred to a chest clinic. Ann Med 1989; 21: 425-427.

25 O'Connell F, Thomas VE, Pride NB, Fuller RW. Capsaicin cough sensitivity decreases with successful treatment of chronic cough. Am J Respir Crit Care Med 1994; 150: 374-380.

26 Birring SS, Prudon B, Carr AJ, Singh SJ, Morgan MDL, Pavord ID. Development of a symptom specific health status measure for patients with chronic cough: Leicester Cough Questionnaire (LCQ). Thorax 2003; 58: 339-343.

27 Kastelik JA, Ojoo JC, Thompson RH, Mulrennan S, Redington AE, Morice AH. Successful treatment of chronic cough is associated with improvement in health status. Am J Respir Crit Care Med 2003; 167: A314.

28 Gibson PG, Dolovich J, Denburg J, Ramsdale EH, Hargreave FE. Chronic cough: eosinophilic bronchitis without asthma. Lancet 1989; 1: 1346-1348.

29 Gibson PG, Hargreave FE, Girgis-Gabardo A, Morris M, Denburg JA, Dolovich J. Chronic cough with eosinophilic bronchitis: examination for variable airflow obstruction and response to corticosteroid. Clin Exp Allergy 1995; 25: 127-132.

30 Mcleod RL, Mingo G, O'Reilly S, Ruck LA, Bolser DC, Hey JA. Antitussive action of antihistamines is independent of sedative and ventilation activity in the guinea pig. Pharmacology 1998; 57: 57-64.

31 Leynaert B, Bousquet J, Neukirch C, Liard R, Neukirch F. Perennial rhinitis: an independent risk factor for asthma in nonatopic subjects: results from the European Community Respiratory Health Survey. J Allergy Clin Immunol 1999; 104: 301-304. 
32 Braman SS, Barrows AA, DeCotiis BA, Settipane G, Corrao WM. Airway hyperresponsiveness in allergic rhinitis. A risk factor for asthma. Chest 1987; 91: 671674.

33 Chakir J, Laviolette M, Turcotte H, Boutet M, Boulet L-P. Cytokine expression in the lower airways of nonasthmatic subjects with allergic rhinitis: influence of natural allergen exposure. J Allergy Clin Immunol 2000; 106: 904-910.

34 Taytard A, Beaumont D, Pujet JC, Sapene M, Lewis PJ. Treatment of bronchial asthma with terfenadine; a randomized controlled trial. Br J Clin Pharmacol 1987; 24: 743-746.

35 Rafferty P, Jackson L, Smith R, Holgate ST. Terfenadine, a potent histamine $\mathrm{H}_{1}$-receptor antagonist in the treatment of grass pollen sensitive asthma. Br J Clin Pharmacol 1990; 30: 229-235.
36 Kastelik JA, Thompson RH, Aziz I, Ojoo JC, Redington AE, Morice AH. Sex-related differences in cough reflex sensitivity in patients with chronic cough. Am J Respir Crit Care Med 2002; 166: 961-964.

37 Fujimura M, Sakamoto S, Kamio Y, Matsuda T. Sex difference in the inhaled tartaric acid cough threshold in non-atopic healthy subjects. Thorax 1990; 45: 633-634.

38 Dicpinigaitis PV, Rauf K. The influence of gender on cough reflex sensitivity. Chest 1998; 113: 1319-1321.

39 Gibson GR. Enalapril-induced cough. Arch Intern Med 1989; 149: 2701-2703.

40 Chang AB, Phelan PD, Sawyer SM, Bel Brocco S, Robertson CF. Cough sensitivity in children with asthma, recurrent cough, and cystic fibrosis. Arch Dis Child 1997; 77: 331-334. 